University of Nebraska - Lincoln

DigitalCommons@University of Nebraska - Lincoln

\title{
Arterial Wall Mechanics and Clinical Implications after Coronary Stenting: Comparisons of Three Stent Designs
}

\author{
Linxia Gu \\ University of Nebraska-Lincoln, gul@fit.edu \\ Shijia Zhao \\ University of Nebraska-Lincoln \\ Stacey R. Froemming \\ Children's Hospital and Medical Center, Omaha, NE
}

Follow this and additional works at: https://digitalcommons.unl.edu/mechengfacpub

Part of the Mechanical Engineering Commons

Gu, Linxia; Zhao, Shijia; and Froemming, Stacey R., "Arterial Wall Mechanics and Clinical Implications after Coronary Stenting: Comparisons of Three Stent Designs" (2012). Mechanical \& Materials Engineering Faculty Publications. 67.

https://digitalcommons.unl.edu/mechengfacpub/67

This Article is brought to you for free and open access by the Mechanical \& Materials Engineering, Department of at DigitalCommons@University of Nebraska - Lincoln. It has been accepted for inclusion in Mechanical \& Materials Engineering Faculty Publications by an authorized administrator of DigitalCommons@University of Nebraska Lincoln. 
Published in International Journal of Applied Mechanics 4:2 (2012), 1250013 (14 pages);

doi: $10.1142 / S 1758825112500135$

http:/ / www.worldscientific.com/doi/abs/10.1142/S1758825112500135

Copyright (C) 2012 Imperial College Press. Used by permission.

Submitted October 24, 2011; accepted February 21, 2012; published June 30, 2012.

\title{
Arterial Wall Mechanics and Clinical Implications after Coronary Stenting: Comparisons of Three Stent Designs
}

\author{
Linxia Gu, ${ }^{1,2}$ Shijia Zhao, ${ }^{1}$ and Stacey R. Froemming ${ }^{3}$
}

1. Department of Mechanical and Materials Engineering University of Nebraska-Lincoln Lincoln, NE 68588-0656, USA

2. Nebraska Center for Materials and Nanoscience Lincoln, NE 68588-0656, USA

3. Hybrid Catheterization and Electrophysiology Laboratory Children's Hospital and Medical Center Omaha, NE 68114-4133, USA

Corresponding author _ L.Gu, 1gu2@unl.edu

\begin{abstract}
The goal of this work is to quantitatively assess the relationship between the reported restenosis rates and stent induced arterial stress or strain parameters through finite element method. The impact of three stent designs (Palmaz-Schatz stent, Express stent, and Multilink Vision stent) on the arterial stress distributions were characterized. The influences of initial stent deployment location, stent-tissue friction, and plaque properties on the arterial stresses were also investigated. Higher arterial stresses were observed at the proximal end of the plaque. The Multilink-Vision stent induced lesser stress concentrations due to the high stiffness of the Cobalt Chromium material and thinner strut thickness. The stentinduced arterial stress concentrations were positively correlated with the reported in-stent restenosis rates, with a correlation coefficient of 0.992 . Stent deployment initiated at the center of the lumen led to less arterial stress variation, while deployment closer to the thinner edge of the plaque causes higher arterial stresses. The friction between the stent and tissue was found to contribute to larger stress alternations for the plaque only. Increased plaque stiffness resulted in a reduced arterial stress concentration and clinical restenosis rate. Results presented herein suggested that arterial stresses serve as a comprehensive index factor to predict the occurrence of in-stent restenosis, which will facilitate the new stent design and surgical planning.
\end{abstract}

\section{Keywords}

In-stent restenosis, correlation, friction, operator, plaque, finite element analysis 


\section{Introduction}

Stent implantation is one of the most common minimally invasive treatments for opening the obstructed coronary artery. The increase in the popularity of stenting as a primary treatment and extension of stenting techniques to more complex lesion types has prompted innovative and competitive stent designs [Oesterle et al., 1998; Colombo et al., 2002;Mackerle, 2005]. A stent is a mesh structure used to restore the patency in stenosed arteries and provide a permanent scaffold for arterial walls. The permanent implanted stent subjects the artery to abnormal stresses which may lead to the occurrence of in-stent restenosis, a major complication of stenting [Oesterle et al., 1998]. Clinical trials have reported various restenosis rates on different stent designs [Becker, 1991; Oesterle et al., 1998; Schwartz et al., 1999; Squire et al., 1999; Kastrati et al., 2000; Palmaz, 2002; Steinman et al., 2003]. Morton has performed a clinical review on the impact of a plethora of physical stent parameters, such as the stent length, diameter, percent metal coverage, strut density, strut thickness, surface quality, cross-section shape, symmetry of deployment, and stent material [Morton et al., 2004]. It is speculated that considerable strain on the vessel wall imposed by the stent or the balloon of the catheter on the endothelium have caused the production of smooth muscle cell abundance and fibroblast growth factors, which led to the restenosis. Understanding the impact of mechanical factors on the arterial injury is essential to develop strategies to prevent and treat a major source of stent failure like restenosis.

Finite element analysis has been proven to be a useful and efficient tool for the study of stent expansion, the interaction between the stent and catheter balloon, and stent-plaque-artery interactions. Cui et al. studied the influence of balloon length and compliance on the mechanical behaviors of expanded stent, such as foreshortening, dogboning and stent recoil [Cui et al., 2010]. De Beule has summarized the computational modeling of balloon-expandable stents, which did not include the interaction between the stent and balloon [De Beule, 2009]. Auricchio et al. studied the biomechanical interaction between a stent and a stenosed artery [Auricchio et al., 2001]. Wang et al. examined the stresses in the artery wall near the ends of the stent [Wang et al., 2006]. Bedoya et al. suggested that stents with small strut spacing (the link between two struts), zero radius of curvature at the end of links will cause high stresses over larger areas of the artery [Bedoya et al., 2006]. According to the best available data, the effects of friction and stent deployment location have not been reported in the numerical studies. Quantitative correlation between arterial stress parameters with the occurrence of in-stent restenosis is also lacking.

In this work, stress distributions on the arterial wall was determined and the clinical implications associated with stenting was investigated by testing the hypothesis that different stent designs will provoke different level of stress concentration in the artery, which are correlated with the occurrence rate of instent restenosis. Three commercially available balloon expandable stent designs (Palmaz-Schatz stent, Express stent, and Multilink-Vision stent) were modeled 
to compare mechanical characteristics of stented arteries using the commercial software ABAQUS (Dassault Syst emes Simulia Corp., Providence, RI, USA). The impact of friction between stent and tissue was evaluated. The stent deployment location inside the lumen was investigated to estimate the influence of the clinical operator. Three stages of plaques (Cellular, Hypo-cellular, and Calcified) were also used to assess the significance of the material properties.

\section{Finite Element Modeling}

A segment of proximal left anterior descending coronary artery (LAD) before the origin of the first septal branch is studied, which is represented by a straight cylinder with a $3 \mathrm{~mm}$ lumen diameter and a length of $26 \mathrm{~mm}$. The thickness of the artery is assumed as $1 / 4$ th of the lumen, i.e., $0.75 \mathrm{~mm}$.

The asymmetric plaque has a parabolic longitudinal profile (Figure 1). An edge ratio of 2:1 at the narrowest lumen leads to a remaining lumen diameter of $50 \%$ of the reference lumen, which is referred to as $50 \%$ stenosis. The longitudinal length of the plaque is $13 \mathrm{~mm}$. Both the plaque and artery are expanded to the reference lumen size of $3 \mathrm{~mm}$ by three types of stents (Figure 2). The physical parameters and restenosis rates for these stents are summarized in Table 1.
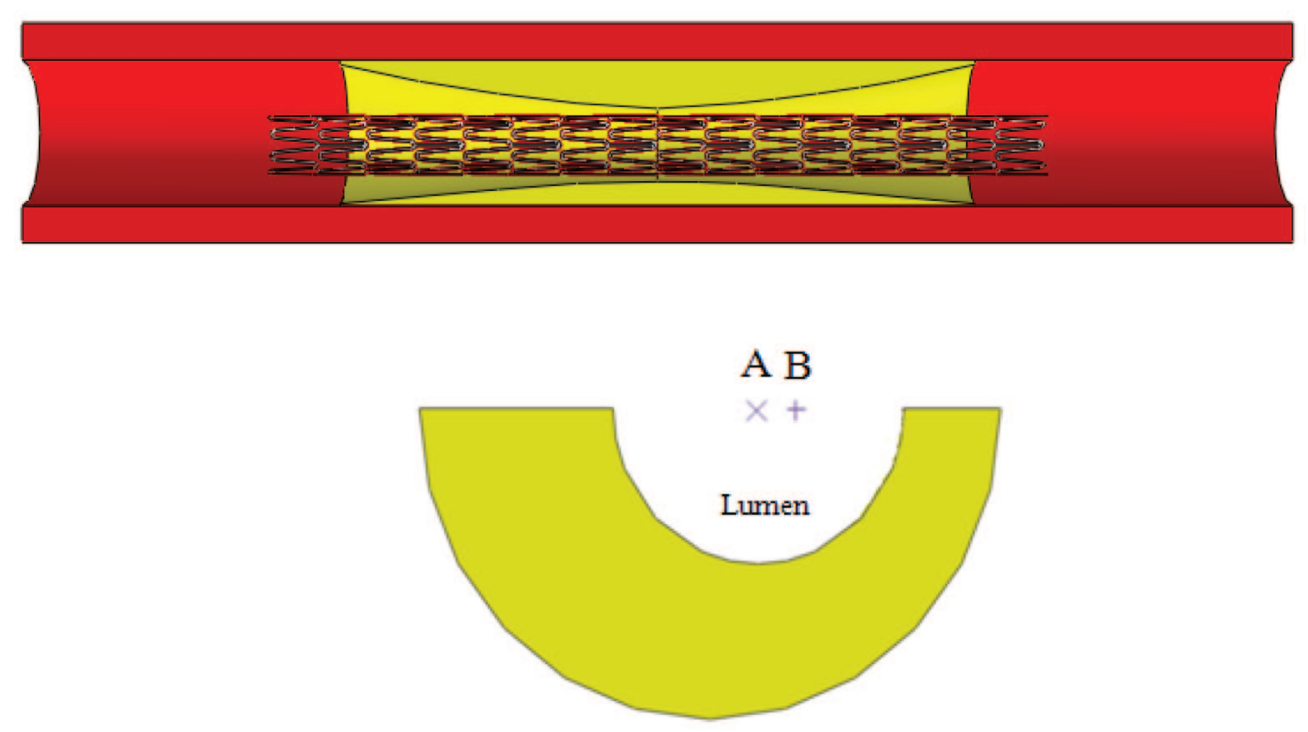

Figure 1. Top: an asymmetric stenosed coronary artery segment with a crimped Express stent inside. Bottom: the cross-section of half-plaque marked with A as center of lumen, and $B$ as $0.1 \mathrm{~mm}$ away from A toward the thinner edge. 


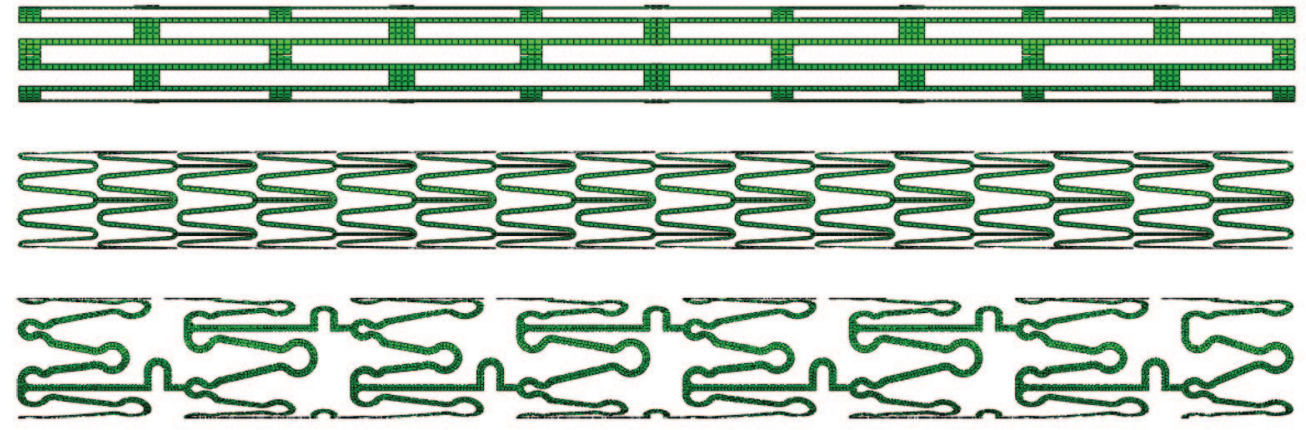

Figure 2. Three representative stents. Top: Palmaz-Schatz (PS) stent. Middle: Express stent. Bottom: Multilink-Vision stent.

Table 1. Physical parameters of three stents and the reported restenosis rates.

\begin{tabular}{|c|c|c|c|}
\hline & $\begin{array}{l}\text { Palmaz-Schatz stent } \\
\text { (Johnson \& Johnson) }\end{array}$ & $\begin{array}{c}\text { Express Stent } \\
\text { (Boston Scientific) }\end{array}$ & $\begin{array}{l}\text { Multilink-Vision } \\
\text { stent (Guidant) }\end{array}$ \\
\hline Metal/Artery area ratio & $30.77 \%$ & $27.12 \%$ & $24.80 \%$ \\
\hline Material & 316LN Stainless steel & 316LN Stainless steel & $\begin{array}{l}\text { L605 Cobalt } \\
\text { Chromium }\end{array}$ \\
\hline Length & $16 \mathrm{~mm}$ & $16 \mathrm{~mm}$ & $15.34 \mathrm{~mm}$ \\
\hline Outer diameter & $1.2 \mathrm{~mm}$ & $1.2 \mathrm{~mm}$ & $1.42 \mathrm{~mm}$ \\
\hline Strut width & 0.07 & 0.05 & 0.08 \\
\hline Strut thickness & $0.09 \mathrm{~mm}$ & $0.13 \mathrm{~mm}$ & $0.08 \mathrm{~mm}$ \\
\hline $\begin{array}{l}\text { No. of units along } \\
\text { circumferential }\end{array}$ & 6 & 8 & 3 \\
\hline $\begin{array}{l}\text { 6-month restenosis } \\
\text { rate }\end{array}$ & $\begin{array}{l}\text { 31.6\% [Fischman } \\
\text { et al., 1994] }\end{array}$ & $\begin{array}{c}\text { 19\% [FDA approval } \\
\text { P020009 September } \\
11,2002]\end{array}$ & $\begin{array}{c}\text { 15.7\% [FDA approval } \\
\text { P020047 July } \\
16,2003 \text { ] }\end{array}$ \\
\hline
\end{tabular}

\subsection{Material models}

The metallic stents are modeled as perfect linear elastic-plastic materials. The material properties for 316LN stainless steel are as follows: Young's modulus E $=190 \mathrm{GPa}$; Poisson ratio $v=0.3$; Yield stress $\sigma_{Y}=207 \mathrm{MPa}$; Limit stress $\sigma_{M}=515$ MPa; Limit nominal strain $\varepsilon_{M}=60 \%$. The plastic behavior of stents is modeled assuming isotropic hardening, which was validated by Auricchio et al. [2001]. The material properties for L605 Cobalt Chromium alloy are as follows: Young's modulus E = $243 \mathrm{GPa}$; Poisson ratio $v=0.3$; Yield stress $\sigma_{Y}=500 \mathrm{MPa}$; Limit stress $\sigma_{M}=1000 \mathrm{MPa}$ [Poncin et al., 2004].

Both artery and plaque are defined by a hyperelastic isotropic constitutive model. The 3rd-order polynomial form of the strain energy density function $U$ is used in this work.

$$
\begin{aligned}
U= & C_{10}\left(I_{1}-3\right)+C_{01}\left(I_{2}-3\right)+C_{11}\left(I_{1}-3\right)\left(I_{2}-3\right)+C_{20}\left(I_{1}-3\right)^{2} \\
& +C_{02}\left(I_{2}-3\right)^{2}+C_{30}\left(I_{1}-3\right)^{3}+C_{03}\left(I_{2}-3\right)^{3} \\
& +C_{12}\left(I_{1}-3\right)\left(I_{2}-3\right)^{2}+C_{21}\left(I_{1}-3\right)^{2}\left(I_{2}-3\right)
\end{aligned}
$$


where $C_{i j}$ are material coefficients determined from the experimental data, while $I_{1}$ and $I_{2}$ are the first and second invariants of the Cauchy-Green tensor, respectively. $I_{1}$ and $I_{2}$ are the strain invariants and

$$
I_{1}=\lambda_{1}^{2}+\lambda_{2}^{2}+\lambda_{3}^{2} ; \quad I_{2}=\lambda_{1}^{-2}+\lambda_{2}^{-2}+\lambda_{3}^{-2}
$$

where $\lambda_{i}$ are the principal stretches.

Uniaxial tension testing has been conducted in a laboratory setting on fresh porcine pulmonary artery [Zhao et al., 2010]. The fitted material coefficients, $C_{10}$ $=0.014881, C_{20}=0.014920$, and $C_{30}=0.00093934$, are used for the arterial material in this work (Figure 3). Due to unavailability of plaque material, its material properties are adopted from published data [Auricchio et al., 2001; Pericevic et al., 2009]. Four plaque models including cellular, hypocellular and calcified tissues are summarized in Table 2. It is clear that the reference plaque is the softest material (Figure 3).

Table 2. Hyperelastic material coefficients for plaque (Unit: MPa).

\begin{tabular}{lcclllll}
\hline Plaque & \multicolumn{1}{c}{$C_{10}$} & $C_{01}$ & $C_{11}$ & $C_{20}$ & $C_{02}$ & $C_{30}$ & \multicolumn{1}{c}{$C_{03}$} \\
\hline Reference & 0.04 & & & & 0.003 & & 0.02976 \\
Cellular & -0.80272 & 0.831636 & 1.15768 & 0 & & 0 & \\
Hypocellular & 0.165111 & 0.016966 & 0.955388 & 0 & & 0 & \\
Calcified & -0.49596 & 0.50661 & 1.19353 & 3.6378 & & 4.73725 & \\
\hline
\end{tabular}

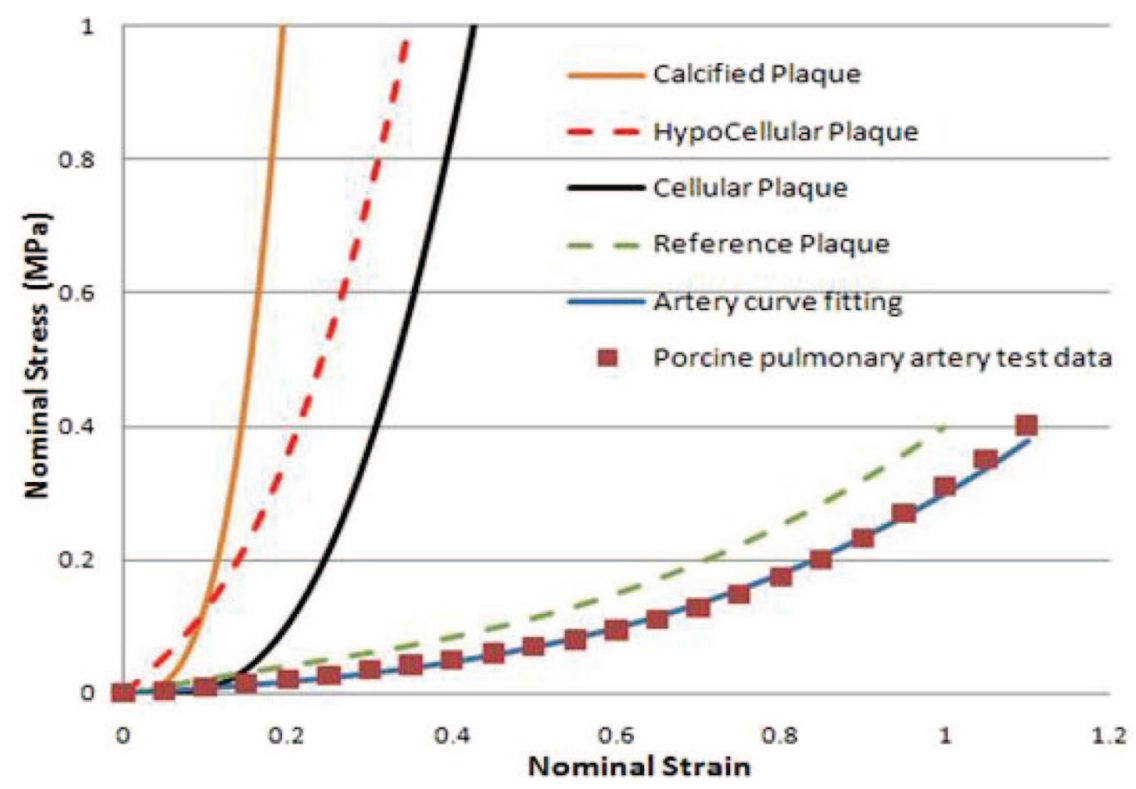

Figure 3. Mechanical behavior of tissue. 


\subsection{Mesh and boundary conditions}

The FE model was constructed to simulate the stent expansion in the stenosed artery and to predict the stresses and strains in the artery wall after the stent expansion. The three-dimensional geometries of stents were developed using the commercial software SolidWorks (Dassault Systèmes SolidWorks Corp., Concord, MA, USA) and then subsequently imported into ABAQUS to perform nonlinear large deformation analysis. The stent was meshed with four-node general shell elements S4R that account for transverse shear stresses and strains. Reduced Hybrid eight-node brick elements C3D8RH were used for the plaque and artery. The stent model was validated by our previous in vitro test [Gu et al., 2010]. The mesh size was chosen as $0.12 \mathrm{~mm}$ and $0.14 \mathrm{~mm}$ for plaque and artery, respectively through mesh convergence studies.

A uniform displacement was applied on the internal surface of the stent until the final diameter of the stent reached $3 \mathrm{~mm}$, which is corresponding to an equivalent uniform pressure loading of 10.5 atm for PS stent. The axial displacement and rotational degree of freedom along radial and circumferential directions are constrained at both ends of the artery to simulate the connections to the other parts of the vasculature. A finite sliding surface to surface contact between stent, plaque and artery was prescribed. The finite sliding approach is the most general tracking approach and allows for arbitrary relative separation, sliding and rotation of the contact surfaces. A friction coefficient of 0.05 is adopted at the tissue-stent interface based on the measurements conducted by Dunn et al. [2007]. The initial stress of the stent and artery were neglected. The influences of three stent designs, stent-artery friction, stent deployment location, and plaque properties on the stresses developed in the arterial wall are investigated.

\section{Results}

\subsection{The effect of stent designs}

All stents were uniformly expanded from their crimping diameters $(1.2 \mathrm{~mm}$ for PS and Express stents, $1.42 \mathrm{~mm}$ for Multilink-Vision stent) to $3.0 \mathrm{~mm}$ inside a 50\% stenosed artery. The stent designs caused deviations in arterial stress. On the arterial wall, the Von Mises (VM) stresses are higher at the proximal region of the plaque (Figure 4). The maximum VM stress was $0.1031 \mathrm{MPa}$ for the PS stent, $0.1123 \mathrm{MPa}$ for the Express stent, and $0.1079 \mathrm{MPa}$ for the Multilink-Vision stent. The maximum principal logarithm strain imparted by the PS stent, Express stent, and Multilink-Vision stent was 0.409, 0.420, and 0.405, respectively. This indicates that mechanical environment of the artery was altered most by the Express stent. While all stents were subjected to the same linear ramping displacement, the maximum principal strain rate (strain versus time) on the stented arterial wall was $2.2281 / \mathrm{s}$ for the PS stent, 1.1141 /s for the Express stent, and 0.9001 /s for the Multilink-Vision stent, respectively. 

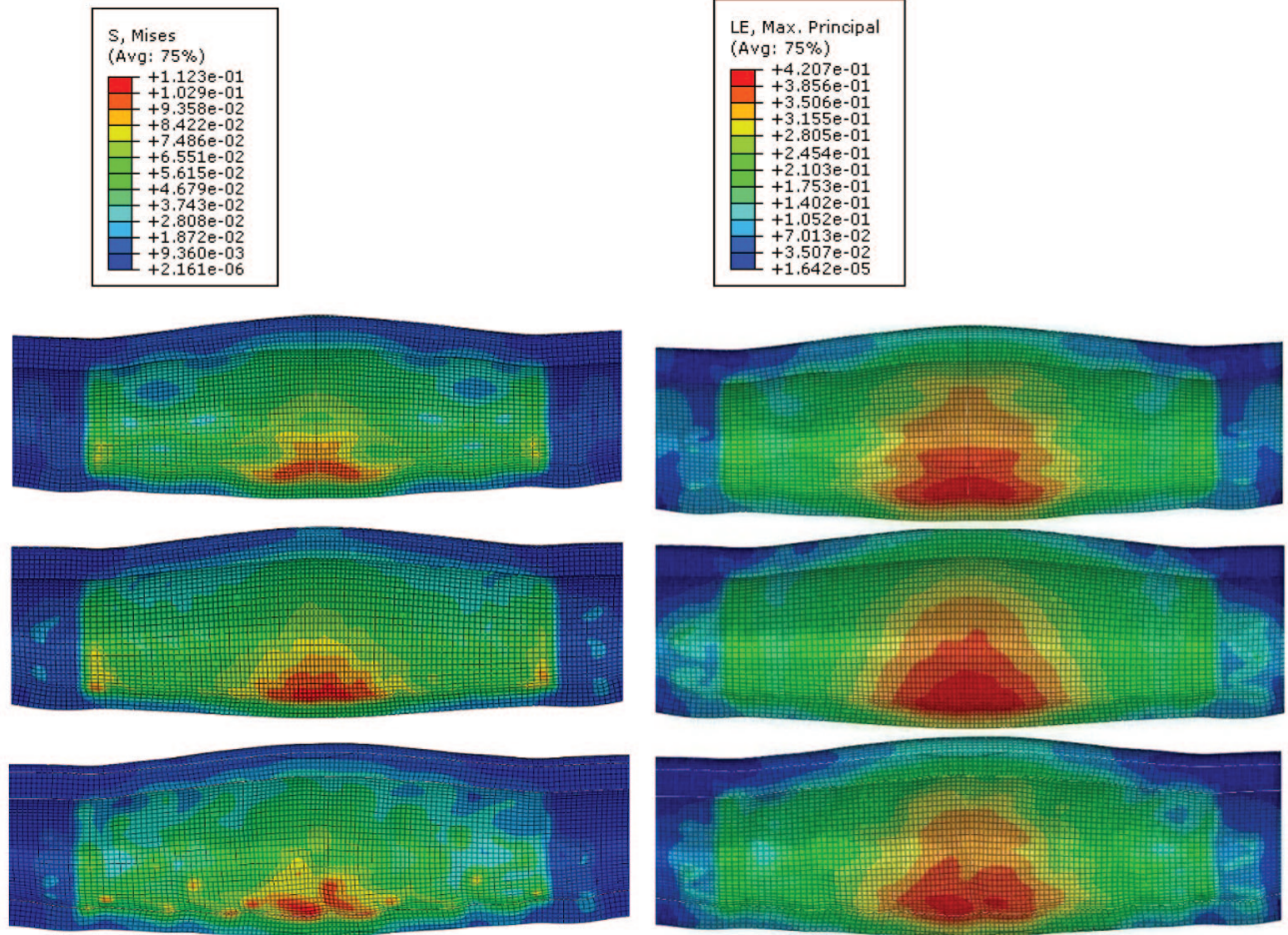

Figure 4. Von Mises stress distribution (Left) and maximum principal logarithm strain distribution (Right) on stented arterial wall: (top) PS stent, (middle) Express stent, and (bottom) Multilink-Vision stent.

While all stents were expanded to a $3 \mathrm{~mm}$ diameter, the Express stent induced higher stresses over larger areas of the arterial wall, as demonstrated in the Figure 5. The percentage arterial VM stress area above the normal physiological stress value $(0.08 \mathrm{MPa})$ was $3.56 \%$ for the Express stent. This elevated stress region accounted for $2.69 \%$ of arterial area for PS stent and $1.64 \%$ for the MultilinkVision stent. This indicates that a thinner strut, i.e., the Multilink-Vision stent in this work, evenly distributes the metal coverage area on the interface, and therefore benefits the host artery in terms of mechanical stress. This agrees with other clinical observations [Morton et al., 2004; Zahedmanesh et al., 2009].

The stress and stress gradient developed in the artery along the longitudinal path under the thinnest plaque edge, the path with higher local stresses, are depicted in Figure 6. Elevated VM stresses were found in the distal end of the plaque. The stress gradient is largest for the Express stent $(0.214 \mathrm{MPa} / \mathrm{mm})$, followed by the Multilink-Vision stent $(0.166 \mathrm{MPa} / \mathrm{mm})$ and the PS stent $(0.160$ $\mathrm{MPa} / \mathrm{mm})$. This may be due to the larger compliance mismatch for the Multilink-Vision stent. The compliance $(\Delta p / \Delta r)$ is $0.56 \mathrm{MPa} / \mathrm{mm}$ for the PS stent implanted in the stented artery. However, a much higher compliance of $0.68 \mathrm{MPa}$ / $\mathrm{mm}$ is observed for the Express stent after its implantation, which is much higher than the artery compliance of $0.046 \mathrm{MPa} / \mathrm{mm}$. 


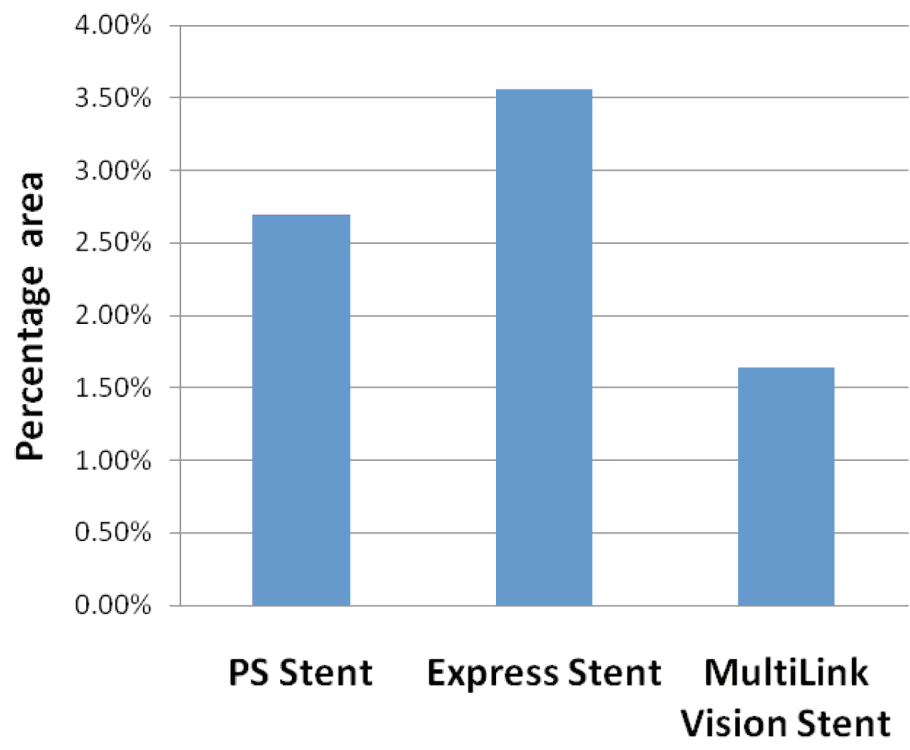

Figure 5. Percentage area of the stented arterial wall area with VM stress $>0.08 \mathrm{MPa}$.

\subsection{The correlation between stress concentration and restenosis rate}

Clinical trials showed that the occurrence of restenosis for the PS stent is $31.6 \%$ when used in 70\% stenosed vessels [Fischman et al., 1994], 19\% for the Express stent deployed in 50\% stenosed vessels (FDA approval P020009 September 11, 2002), and $15.7 \%$ for the Multilink-Vision stent in $50 \%$ stenosed vessels (FDA approval P020047 July 16, 2003). The plaque in the previous PS stent-plaque-artery interaction model is revised to construct a $70 \%$ stenosis. The 2:1 unsymmetrical edge ratio remained. The maximum VM stress on the artery wall after PS stent deployment increased from $0.1031 \mathrm{MPa}$ for a $50 \%$ stenosed vessel to $0.163 \mathrm{MPa}$ for a $70 \%$ stenosed vessel. The stenosis level caused a very significant stress increase of $58 \%$ in the arterial wall, indicating a higher potential for injury.

The relationship between the maximum VM stresses and the reported restenosis rates was illustrated in Figure 7. It is clear that higher stress indicates a higher restenosis rate. The Pearson correlation coefficient between these two datasets (i.e., restenosis rate and the maximum VM stress for various stent designs) is 0.992 . The Pearson correlation coefficient of two sets of data is calculated by

$$
r=\frac{\sum_{i=1}^{n}\left(x_{i}-\bar{x}\right) *\left(y_{i}-\bar{y}\right)}{\sqrt{\sum_{i=1}^{n}\left(x_{i}-\bar{x}\right)^{2} \sum_{i=1}^{n}\left(y_{i}-\bar{y}\right)^{2}}}
$$

where $\bar{x}$ and $\bar{y}$ are average value of each dataset. Correlation coefficient $r$ is in the range of $[-1,1]$. A coefficient closer to one indicates a stronger correlation be- 

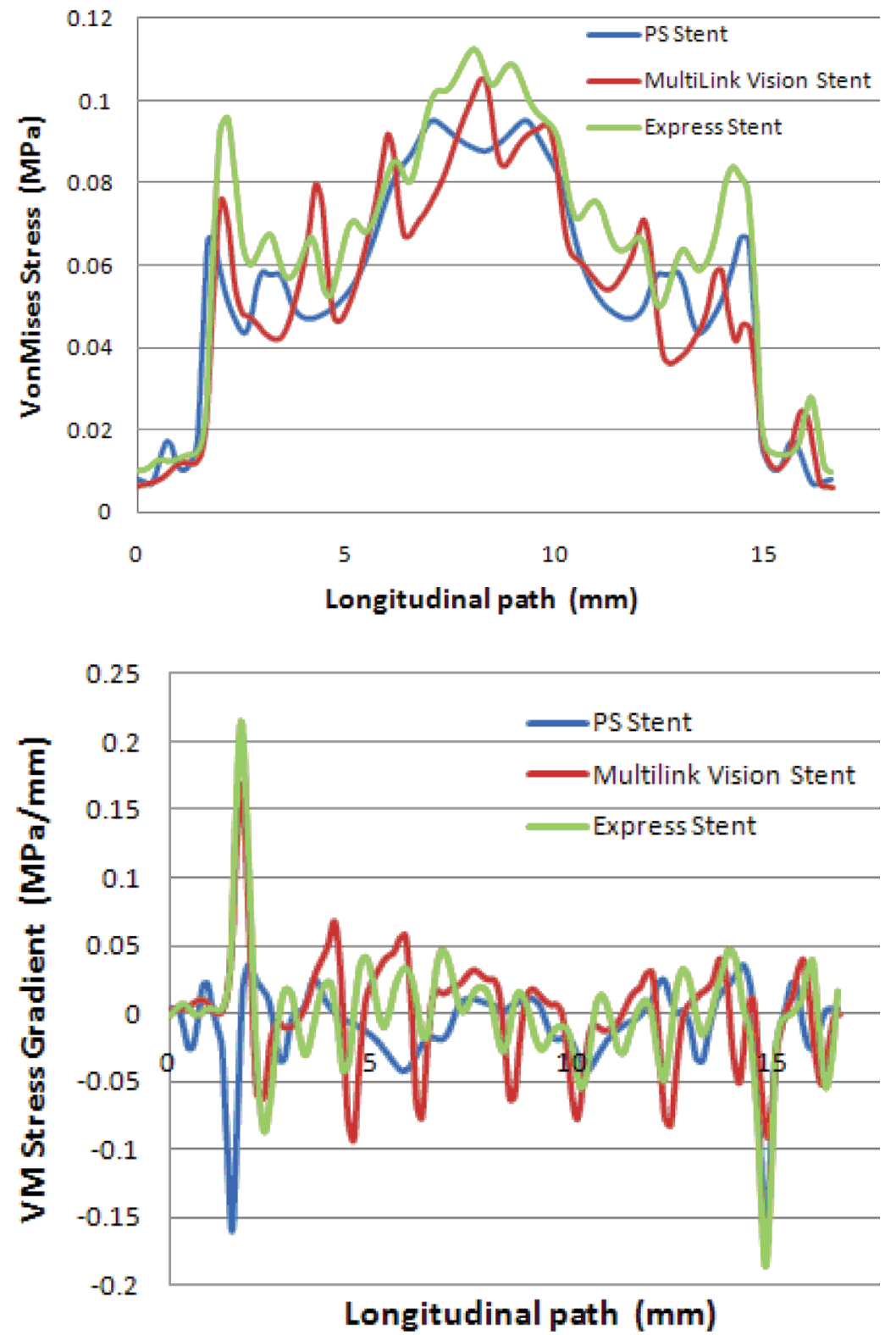

Figure 6. Comparison of Von Mises stress (top) and stress gradient (bottom) on the inner surface of the stented artery under thinner plaque profile.

tween datasets. The significant correlation $(r=0.992)$ between the maximum VM stresses and the reported restenosis rates validated our hypothesis that various stent designs will provoke different levels of stresses in the artery, predicting the occurrence of restenosis very well. 


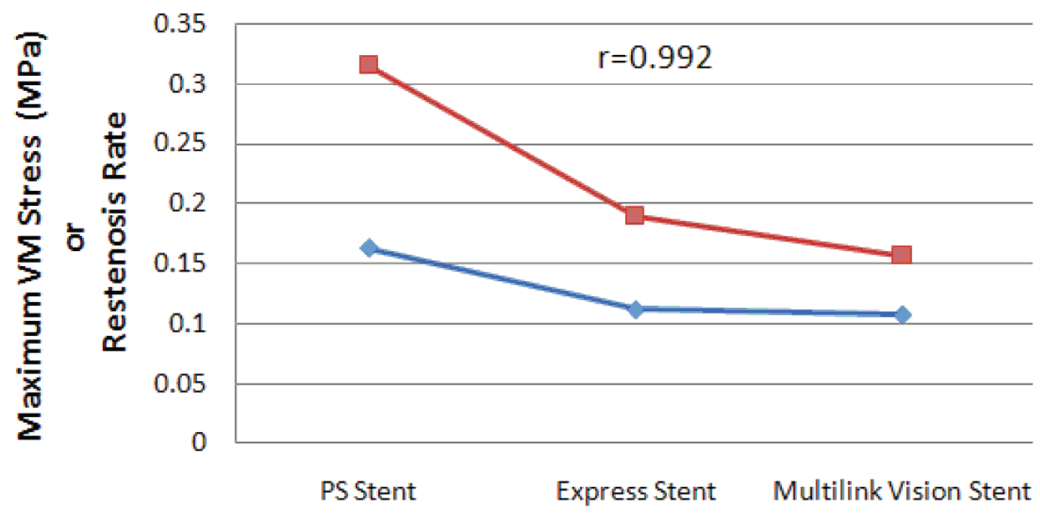

Figure 7. Correlation between the maximum VM stresses and the reported restenosis rates.

\subsection{The effect of deployment location}

The stent is assumed to be deployed from the center of the lumen at the narrowest lesion site. To assess the impact of the operator skills, we changed the stent deployment from the center location, marked as point A in Figure 1, to 0.1 $\mathrm{mm}$ toward the thinner edge of plaque for the PS stent, i.e., the stent is closer to the thinner edge of the plaque before it expands, as demonstrated as point B in Figure 1. The maximum VM stress in the stented artery was then elevated $12.7 \%$. The effect of the deployment location was summarized in Table 3, which reveals that the uncertainty in the initial deployment location affects the complications of the stenting procedure.

\subsection{The effect of friction on the stent-tissue interface and plaque properties}

The frictionless contact between stent and tissue was used in the previous comparison of the performance of all stents. The relative motion between stent and endothelial cells during deployment was quantified by Dunn et al. [2007]. They suggested a friction coefficient in the range of 0.03 to 0.06 . In this work, a friction coefficient of 0.05 was applied on the stent-tissue interfaces. The maximum VM stress on the plaque increased by $15.32 \%$ due to the existence of friction. However, the stress on the artery only changed by $1.4 \%$. The variations of stress and strain in the arterial wall were much less for the calcified plaque. This indicates that the arterial stress field is not much affected by the friction between surfaces.

Table 3. Results for different stent initial positions.

\begin{tabular}{lcccc}
\hline $\begin{array}{l}\text { Deployment } \\
\text { location }\end{array}$ & $\begin{array}{c}\text { 0.1mm toward } \\
\text { thicker edge }\end{array}$ & $\begin{array}{c}\text { Center of } \\
\text { lumen }(\mathrm{A})\end{array}$ & $\begin{array}{c}0.05 \mathrm{~mm} \text { toward } \\
\text { thinner edge }\end{array}$ & $\begin{array}{c}0.1 \mathrm{~mm} \text { toward } \\
\text { thinner edge (B) }\end{array}$ \\
\hline Maximum VM stress & $0.1016 \mathrm{MPa}$ & $0.1031 \mathrm{MPa}$ & $0.1046 \mathrm{MPa}$ & $0.1162 \mathrm{MPa}$ \\
\hline
\end{tabular}


The effect of various stages of plaques (reference plaque, cellular plaque, hypocellular plaque, and calcified plaque, with increasing stiffness) was evaluated for the PS stent. The maximum VM stress was $0.1017 \mathrm{MPa}$ for the reference plaque, $0.3855 \mathrm{MPa}$ for the cellular plaque, $0.2719 \mathrm{MPa}$ for the hypocellular plaque, and $0.2172 \mathrm{MPa}$ for the calcified plaque. This agrees with the speculation of Pericevic et al. [2009]. They suggested that the stiffer plaque will play a protective role on host artery by imparting lower magnitude stresses and, therefore, diminish the risk of injury to the vessel wall for a given inflation pressure.

\section{Discussions and Conclusion}

One of the major complications of stent implantation is restenosis. The risk factors of restenosis are identified as stent design, arterial properties, geometry, and so on [Morton et al., 2004]. Mechanical stress parameter is able to incorporate the above mentioned risk factors and thus serves as a unique comprehensive risk factor to predict in-stent restenosis. In this work, computational models were used to predict the wall mechanics of three stent designs and establish correlations between the obtained arterial wall stress concentrations and the restenosis data from published clinical trials. Although vascular anatomy and physical properties varied case by case, the same properties are adopted here for comparative study of the impact of stent designs.

The subsequent results show that stent designs are indeed the cause of large alternations in the mechanical environment of the arterial wall. The maximum VM stresses on the arterial wall are correlated with the reported restenosis rate with a correlation coefficient of 0.992 , which validated the hypothesis that stent designs will provoke different levels of stress concentration in the artery, which are correlated with the occurrence rate of in-stent restenosis. Therefore, it is important to understand the stresses induced by the stent in the artery wall in order to improve stent design.

Even when all the stents are expanded to the same final dimensions in the same stenosed vessel, the maximum VM stress on the artery is increased $9 \%$ by changing the implanted stent from the PS model to the Express model. The Express stent induced higher stresses over larger areas of the arterial wall, and higher stress concentration in the distal end of the plaque. The maximum VM stress on the artery is increased by $12.7 \%$ when the stent deployment moves closer to the thinner plaque side, indicating the operator's impact on the outcome of stenting. This could be explained by the less resistance from the plaque and severe overstretch of the artery wall. The maximum stress or stain on the arterial wall is dependent on the material properties of the plaque. Stenting induced arterial stresses to increase with an increased cellular component concentration in the plague. As the plaque properties varied from the cellular type to the calcified one, the maximum stress and its gradient on the artery wall reduced almost by $50 \%$ for PS stent implantations. Increased plaque stiffness resulted in a reduced arterial stress concentration, which agrees with the observation by Pericevic et al. [2009]. The friction on the tissue-stent interface altered the plaque mechanics sub- 
stantially, which may lead to the larger deformation for the cellular plaque and even fracture of the calcified plaque. However, the impact of friction on the artery is minimal without considering the damage mechanics. Stress distributions in the artery were significantly affected by the uncertainty of the deployment position of the stent.

In this work, the obtained positive correlation between the arterial stress concentrations and the reported in-stent restenosis rates suggested that reduced arterial stress could improve the clinical outcomes. The deployment of stent closer to the thicker side of the plaque and a relative stiffer plaque led to less stresses on the artery wall, which implied a less complication as in-stent restenosis. The quantification of the above-mentioned relationship could provide insight and implication toward a better understanding of the mechanism of restenosis.

In the present model, a balloon used to inflate the stent was modeled by a controlled displacement. The feasibility of the boundary condition was validated by our previous in vitro testing [Gu et al., 2005]. The stent was modeled by a general shell element, which has been verified by the study on the effects of different element types [Avdeev and Shams, 2010]. The tissue materials are assumed to be isotropic and homogeneous, although the artery and plaque have been shown to be anisotropic and viscoelastic [Loree et al., 1994; Salunke et al., 2001]. The geometry of the artery and plaque was also simplified for this comparative study. More realistic models considering patient specific geometry and nonhomogeneous arterial wall properties will change the stress distribution on the arterial wall. For diffuse plaque and curved artery geometries, the inclusion of balloon dynamics in the models will be more appropriate to study the under-expansion effect of stent procedure [Zahedmanesh et al., 2010; De Beule et al., 2008; Mortier et al., 2008]. Despite these simplifications, the present work demonstrated the importance of stent design in terms of a unique risk factor, the maximum VM stress on the artery, which correlates with the occurrence of in-stent restenosis, and may have significant clinical implications.

Conflict of Interest: None of the authors has any conflict of interest.

Acknowledgments - The authors are grateful for funding from the National Science Foundation under grant Nos. 0926880 and 0811250.

\section{References}

Auricchio, F. et al. [2001] "Finite element analysis of a stenotic artery revascularization through stent insertion," Computer Methods in Biomechanics and Biomedical Engineering 4, 249-263.

Avdeev, I. and Shams, M. [2010] Vascular stents: Coupling full 3-D with reduced-order structural models, IOP Conf. Series: Materials Science and Engineering, Mahdia, Tunisia.

Becker, G. J. [1991] "Intravascular stents. General principles and status of lower-extremity arterial applications," Circulation 83(2), I122-136. 
Bedoya, J. et al. [2006] "Effects of stent design parameters on normal artery wall mechanics," Journal of Biomechanical Engineering 128(5), 757-765.

Colombo, A. et al. [2002] "Selection of coronary stents," Journal of the American College of Cardiology 40(6), 1021-1033.

Cui, F. S. et al. [2010] "Effects of balloon length and compliance on vascular stent expansion," International Journal of Applied Mechanics 2(3), 681-697.

De Beule, M. et al. [2008] "Realistic finite element-based stent design: The impact of balloon folding," Journal of Biomechanics 41(2), 383-389.

De Beule, M. [2009] “Biomechanical modelling of stents: Survey 1997-2007," Advances in Biomedical Engineering, ed. P. Verdonck (Elsevier, The Netherlands, Amsterdam), pp. 61-94.

Dunn, A. et al. [2007] "Macroscopic friction coefficient measurements on living endothelial cells," Tribology Letters 27(2), 233-238.

FDA approval P020009 [September 11, 2002]. Summary of Safety and Effectiveness Data (SSED), Express2 Coronary Stent System Boston Scientific Scimed, Inc.

FDA approval P020047 [July 16, 2003]. Summary of Safety and Effectiveness Data (SSED), Multilink Vision RX Coronary Stent System Guidant Corporation.

Fischman, D. L. et al. [1994] “A randomized comparison of coronary-stent placement and balloon angioplasty in the treatment of coronary-artery disease," New England Journal of Medicine 331(8), 496-501.

Gu, L. et al. [2005] "Finite element analysis of covered microstents," Journal of Biomechanics 38(6), 1221-1227.

Gu, L. et al. [2010] "The relation between the arterial stress and restenosis rate after coronary stenting," Journal of Medical Devices 4(3), 031005.

Kastrati, A. et al. [2000] "Influence of stent design on 1-year outcome after coronary stent placement: A randomized comparison of five stent types in 1,147 unselected patients," Catheterization and Cardiovascular Interventions 50(3), 290-297.

Loree, H. M. et al. [1994] "Static circumferential tangential modulus of human atherosclerotic tissue," Journal of Biomechanics 27(2), 195-204.

Mackerle, J. [2005] "Finite element modelling and simulations in cardiovascular mechanics and cardiology: A bibliography 1993-2004," Computational Methods Biomechanical and Biomedical Engineering 8(2), 59-81.

Mortier, P. et al. [2008] "Numerical study of the uniformity of balloon-expandable stent deployment," Journal of Biomechanical Engineering 130(2), 021018.

Morton, A. C. et al. [2004] "The influence of physical stent parameters upon restenosis," Pathologie Biologie 52(4), 196-205.

Oesterle, S. N. et al. [1998] “The stent decade: 1987 to 1997. Stanford Stent Summit faculty," American Heart Journal 136(4 Pt 1), 578-599.

Palmaz, J. C. [2002] "Influence of stent design on clinical outcome," Minimally Invasive Therapy and Allied Technologies 11(4), 179-183.

Pericevic, I. et al. [2009] "The influence of plaque composition on underlying arterial wall stress during stent expansion: The case for lesion-specific stents," Medical Engineering and Physics 31(4), 428-433.

Poncin, P. et al. [2004] Comparing and optimizing Co-Cr Tubing for Stent Applications Materials Processes for Medical Devices Conference. 
Salunke, N. V. et al. [2001] "Compressive stress-relaxation of human atherosclerotic plaque," Journal of Biomedical Materials Research 55(2), 236-241.

Schwartz, E. A. et al. [1999] "Exposure of human vascular endothelial cells to sustained hydrostatic pressure stimulates proliferation. Involvement of the alphaV integrins," Circular Research 84(3), 315-322.

Squire, J. C. et al. [1999] "Measuring arterial strain induced by endovascular stents," Medical and Biological Engineering and Computing 37(6), 692-698.

Steinman, D. A. et al. [2003] "Computational modeling of arterial biomechanics: Insights into pathogenesis and treatment of vascular disease," Journal of Vascular Surgery 37(5), 1118-1128.

Wang, W. Q. et al. [2006] "Analysis of the transient expansion behavior and design optimization of coronary stents by finite element method," Journal of Biomechanics 39(1), 21-32.

Zahedmanesh, H. et al. [2009] "Determination of the influence of stent strut thickness using the finite element method: Implications for vascular injury and in-stent restenosis," Medical and Biological Engineering and Computing 47, 385-393.

Zahedmanesh, H. et al. [2010] "Simulation of a balloon expandable stent in a realistic coronary artery: Determination of the optimum modeling strategy," Journal of Biomechanics 43(11), 2126-2132.

Zhao, S. J. et al. [2010] Mechanical Behavior of Porcine Pulmonary Artery ASME International Mechanical Engineering Congress and RD\&D Expo. Vancouver, British Columbia. 